\title{
Deep Brain Stimulation Utilizing Dexmedetomidine: A Clinical Report from the University of Miami Miller School of Medicine
}

\author{
Derek B. Covington, Meredith M. Degnan, Yiliam F. Rodriguez-Blanco, Ankeet A. Choxi, \\ Rupa S. Prasad, Jonathan R. Jagid, Thomas M. Fuhrman ${ }^{1}$
}

\begin{abstract}
Background: Deep brain stimulation (DBS) is an increasingly utilized technique to treat symptoms of neurological movement disorders, most commonly, Parkinson's Disease. Patients and surgeons alike appreciate the minimally invasive nature of this procedure, as well as its reversibility.As these surgeries are being performed more often, it is becoming increasingly important to optimize our anesthetic management during these cases. Methods: We conducted a retrospective review of the DBS procedures that have been performed at our institution utilizing monitored anaesthesia care (MAC) via dexmedetomidine infusion to report on the frequency and type of perioperative complications as well as to assess the effectiveness of this technique. Results: A total of I 50 patients and I 74 lead placements were included in this study. Dexmedetomidine was the sole anaesthetic used in $\mathbf{8 5 . 6 \%}$ of cases.The remaining cases used a combination of dexmedetomidine and adjuvant agents.A total of one perioperative complication was found in our series, resulting in a total complication rate percentage per patient of $0.6 \%$. Conclusions: We found very few perioperative complications associated with the use of dexmedetomidine during these challenging cases. With its anxiolytic, sedative, and analgesic properties coupled with preservation of respiration and a short half-life, dexmedetomidine has ideal properties for DBS procedures.
\end{abstract}

Key words: Deep brain stimulation, dexmedetomidine, electrode implantation surgery, monitored anaesthesia care, perioperative complications

\section{INTRODUCTION}

Electrode implantation surgery, also known as deep brain stimulation (DBS), is an increasingly utilised technique to treat symptoms of movement disorders,

Department of Anesthesiology, University of Miami Miller School of Medicine, Miami, FL 33136, 'Bay Pines Veterans Administration Medical Center, Bay Pines, FL 33744, USA

\section{Address for correspondence:}

Dr. Meredith M. Degnan, Department of Anesthesiology,

University of Miami Miller School of Medicine,

1611 NW 12 ${ }^{\text {th }}$ Avenue, Miami, FL 33136, USA.

E-mail: meredith.degnan@jhsmiami.org

\begin{tabular}{|l|l|}
\hline \multicolumn{2}{|c|}{ Access this article online } \\
\hline Quick Response Code: & Website: \\
\hline & www.jnaccjournal.org \\
\cline { 2 - 2 } & \\
\hline & \\
\hline
\end{tabular}

such as Parkinson's disease, recalcitrant depression, highly resistant obsessive-compulsive disorder, chronic pain and intractable seizures..$^{[1-5]}$ Viewed as a minimally invasive procedure, it is favoured secondary to its reversibility, adjustability and capability to be performed bilaterally. ${ }^{[1]}$

The anaesthetic technique is challenging as it must account for patients with varying degrees of functional neurologic disorders, the effects of anaesthetic medications on the microelectrode recordings (MERs), avoidance of hypertension or hypotension, and often the desire of the surgeon for an awake, comfortable and

This is an open access article distributed under the terms of the Creative Commons Attribution-NonCommercial-ShareAlike 3.0 License, which allows others to remix, tweak, and build upon the work non-commercially, as long as the author is credited and the new creations are licensed under the identical terms.

For reprints contact: reprints@medknow.com

How to cite this article: Covington DB, Degnan MM, Rodriguez-Blanco YF, Choxi AA, Prasad RS, Jagid JR, et al. Deep Brain Stimulation Utilizing Dexmedetomidine: A Clinical Report from theUniversity of Miami Miller School of Medicine. J Neuroanaesthesiol Crit Care 2016;3:233-8. 
cooperative patient. ${ }^{[6]}$ Although indications for DBS continue to increase, 'it is clear that no simple anesthetic regimen exists' ${ }^{[7]}$

With its anxiolytic, sedative and analgesic properties coupled with preservation of respiration and a short half-life, dexmedetomidine has ideal properties for these types of procedures. We conducted a retrospective review of the DBS procedures that have been performed at our institution utilising monitored anaesthesia care (MAC) via dexmedetomidine infusion. The primary aim of this study is to assess perioperative complications associated with DBS procedures when dexmedetomidine is used as the primary anaesthetic agent.

\section{METHODS}

After the Institutional Review Board approval, 174 DBS lead placements involving 150 patients at Jackson Memorial Hospital in Miami, FL, USA, were retrospectively reviewed from 2007 to 2011 . Anaesthetic preoperative assessments, anaesthetic intra-operative records, anaesthetic postanaesthesia unit notes, nursing recovery notes, operative notes and discharge summaries were reviewed. All medications administered during the perioperative period were noted, including the dose of dexmedetomidine. Patient demographics were recorded including sex, age, height, weight, smoker/nonsmoker status, current medications, indications for DBS, surgical date, surgical location and co-morbidities.

Perioperative complications were also reviewed and were defined by Khatib et al..$^{[8]}$ Potential cardiac complications included intra- or post-operative myocardial infarction, new onset angina, new onset of congestive heart failure, and 'refractory' systemic arterial hypertension. Respiratory complications were defined as respiratory arrest or severe airway obstruction that required endotracheal intubation, prolonged endotracheal intubation, post-operative acute respiratory distress syndrome, nosocomial pneumonia and aspiration. Neurologic complications included new-onset stroke or transitory ischaemic attack, intra- or post-operative intracranial bleeding and seizures. Psychological/psychiatric complications were defined as confusion syndrome, anxiety, delirium and withdrawal syndromes.

All cases were performed with the standard American Society of Anesthesiologists monitors including noninvasive blood pressure, electrocardiogram, pulse oximetry, skin temperature probe and end-tidal carbon dioxide monitoring. If invasive blood pressure monitoring was indicated, an arterial line was placed. All cases were also performed via MAC with a dexmedetomidine infusion throughout the placement of the deep brain stimulator. The depth of anaesthesia was monitored by the vigilance of haemodynamic parameters and assessing patient comfort throughout the procedure. A bispectral index monitoring system was not used in these patients as it would have obscured the operative field. All patients received subcutaneous scalp infiltration with a local anaesthetic before the incision. As per the anaesthesiologist's clinical judgement, dexmedetomidine infusion was appropriately titrated based on the patient's haemodynamic parameters and comfort level throughout the case. In addition, intermittent intravenous (IV) boluses or IV infusions of vasopressors or anti-hypertensive agents were used to control blood pressure within an acceptable range at the discretion of the anaesthesiologists.

The majority of cases were converted from MAC to general endotracheal anaesthesia (GETA) as per the surgeon's request in order to place the generator in the subcutaneous tissue of the chest after ensuring proper placement and function of the DBS leads. Otherwise, MAC was continued for the entire procedure, and the implantation of the generator was scheduled at a future date.

\section{Main outcome measure}

The main outcome measure was to report on the frequency and type of perioperative complications and to assess the effectiveness of utilising dexmedetomidine during deep brain stimulator surgery. The effectiveness of utilising dexmedetomidine was defined as the ability to perform the surgical case to completion without major adverse effects.

\section{Statistical method}

All statistical analyses were performed with the SPSS software statistical package version 22 (SPSS Inc., Chicago, IL, USA). Frequencies and descriptive statistics were applied to the data, as appropriate.

\section{RESULTS AND MAJOR FINDINGS}

A total of 150 patients and 174 lead placements were included in the study. Table 1 illustrates demographics for our study population including, age, gender, neurologic diagnosis, preexisting co-morbidities and laterality of DBS procedures.

The most common anaesthetic technique for the placement of the DBS leads consisted of an IV infusion of dexmedetomidine as the sole sedative and analgesic agent and was used in 149 procedures (85.6\%). A combination of a dexmedetomidine infusion with fentanyl IV was utilised in 12 cases $(6.9 \%)$ while dexmedetomidine with midazolam IV was utilised in 8 cases $(4.6 \%)$. Three procedures were completed with a combination of dexmedetomidine, fentanyl and midazolam. One case used dexmedetomidine and hydromorphone during 
DBS insertion, and one case utilised dexmedetomidine, remifentanil and midazolam.

The mean infusion dose of dexmedetomidine was $0.59 \mathrm{mcg} / \mathrm{kg} / \mathrm{h}$ with a range of doses from 0.2 to $0.7 \mathrm{mcg} / \mathrm{kg} / \mathrm{h}$. A loading dose of $1.0 \mathrm{mcg} / \mathrm{kg}$ over $10 \mathrm{~min}$ was utilised in 70 cases $(40.2 \%)$ based on anaesthesiologist preference. No statistics were measured to assess for the difference in outcomes between patients receiving a loading dose versus no loading dose.

\section{Table 1: Demographics of population sample and surgical cases}

\begin{tabular}{lc}
\hline & Population sample \\
\hline Age, mean (SD) & $66.4(9.3)$ \\
Gender, $n(\%)$ & $110(63.2)$ \\
Male & \\
Diagnosis, $n(\%)$ & $140(80.5)$ \\
Parkinson's disease & $32(18.4)$ \\
Essential tremor & $2(1.1)$ \\
Focal dystonia & \\
Co-morbidities, $n(\%)$ & $76(42.2)$ \\
Hypertension & $49(27.2)$ \\
Cigarette smoking & $40(23.0)$ \\
Obesity & $29(16.1)$ \\
Diabetes & $22(12.2)$ \\
Coronary artery disease & $11(6.1)$ \\
Asthma & $8(4.4)$ \\
COPD & $76(43.7)$ \\
Bilateral DBS procedures, $n(\%)$ & \\
\hline SD=Standard deviation, DBS=Deep brain stimulator, COPD Chronic \\
obstructive pulmonary disorder
\end{tabular}

Treatment with antihypertensive medications was utilised in 134 cases $(77.01 \%)$ while treatment with medications for hypotension was utilised in 70 cases $(40.23 \%)$.

GETA was used in 88 cases (50.6\%) for internal pulse generator insertion after placing the DBS electrodes if the permanent generator was inserted the same day after electrode placement. Except for one case with a laryngeal mask airway, the airway was secured with a cuffed endotracheal tube. The remaining 86 cases $(49.4 \%)$ were completed as MAC with generator placement scheduled to occur at a future surgical date [Table 2].

A total of one perioperative complication was found in our series, resulting in a total complication rate percentage per patient of 0.6. This complication was secondary to a patient becoming severely agitated and uncooperative intraoperatively, which resulted in only a unilateral device placement, instead of the planned bilateral procedure. This patient received no other medications besides an infusion of dexmedetomidine at a rate of $0.3-0.6 \mathrm{mcg} / \mathrm{kg} / \mathrm{h}$ during the procedure.

\section{DISCUSSION}

DBS represents an effective treatment for patients with a variety of neurologic and mood disorders. The anaesthetic management for these procedures can be challenging secondary to a multitude of factors. Preoperatively, patients may suffer from 'off-period' pain, dystonia or mood derangements secondary to the cessation of medications before the surgery. Intraoperatively, patients must be arousable, cooperative and comfortable in the light of frequent comorbidities

Table 2: Intra-operative anaesthetic management

\begin{tabular}{lc}
\hline Anesthetic management technique & $N(\%)$ \\
\hline Cases with dexmedetomidine as sole sedative/analgesic medication during DBS & $149(85.6)$ \\
insertion, $n(\%)$ & $12(6.9)$ \\
Cases with dexmedetomidine and fentanyl during DBS insertion & $8(4.6)$ \\
Cases with dexmedetomidine and midazolam during DBS insertion & $3(1.7)$ \\
Cases with dexmedetomidine, fentanyl and midazolam during DBS insertion & $1(0.6)$ \\
Cases with dexmedetomidine, remifentanil and midazolam during DBS insertion & $1(0.6)$ \\
Cases with dexmedetomidine and hydromorphone during DBS insertion & 0.59 \\
Dexmedetomidine dose (mcg/kg/h), mean & $0.2-0.7$ \\
Dexmedetomidine doses (mcg/kg/h), range & $70(40.2)$ \\
Cases with dexmedetomidine loading dose & $134(77.01)$ \\
Cases requiring treatment with medications for hypertension & $70(40.23)$ \\
Cases requiring treatment with medications for hypotension & $88(50.6)$ \\
MAC cases & $86(49.4)$ \\
Cases converted from MAC to GETA
\end{tabular}

MAC=Monitored anaesthesia care, GETA=General endotracheal anaesthesia, DBS=Deep brain stimulator 
and baseline neurologic deficits. Furthermore, patients are placed in a stereotactic apparatus, which can cause additional pain and anxiety. In addition, airway access may prove difficult in the event of respiratory distress. Postoperatively, patients require vigilant neurologic assessments and should ideally be without the effects of anaesthetics.

Dexmedetomidine represents a unique agent that may fulfil 'the need for a sedative agent with properties that will not interfere with the process of physiologic localization, has little effect on the patient's motor symptomatology or respiratory status, and yet creates an environment in which the patient feels comfortable and relaxes... ${ }^{[8]}$ As an alpha-2 adrenoreceptor agonist, dexmedetomidine has sedative, anxiolytic and analgesic effects. In addition, its short half-life of 6 min allows for rapid wake-up of patients for cognitive testing. ${ }^{[9]}$

We found that a dexmedetomidine infusion during DBS electrode placement provided all of these aforementioned benefits for the patients and the surgeons. In addition, we found very few perioperative complications associated with the use of dexmedetomidine during these challenging cases. The low complication rate found in our study, which was $0.6 \%$, supports the role of dexmedetomidine for usage in DBS surgeries and other similar procedures. In $85.6 \%$ of the cases in this study, dexmedetomidine was the sole anaesthetic agent utilised. If dexmedetomidine alone can be used in the majority of cases, benzodiazepines and opioids, which have numerous and potentially serious side-effects, can be limited or completely avoided. One previous retrospective chart review of 13 DBS procedures and one prospective study of 23 patients also found that dexmedetomidine provided excellent patient comfort and haemodynamic stability with no impairment of intra-operative mapping and minimal respiratory depression. ${ }^{[10,11]}$

Due to our low complication rate, our study was unable to assess risk factors for perioperative complications. However, Khatib et al. previously reported that increasing age was found to be a risk factor in a study of 258 DBS procedures utilising propofol. ${ }^{[8]}$ The authors of this finding postulate that '... the association between age and complications noted in the study could be explained that with increased age the ability of the body to withstand other comorbidities become less and could succumb easily to their effect under lengthy and stressful procedures like DBS. ${ }^{[8]}$ Besides our low complication rate, comparisons between our study and that of Khatib et al. would prove difficult secondary to the different anaesthetic managements. Unlike our review, which utilised an infusion of dexmedetomidine for every case and dexmedetomidine as the sole anaesthetic for the vast majority of cases, more than $90 \%$ of the patients in the review by Khatib et al. received a propofol infusion and only $0.3 \%$ of the patients received dexmedetomidine as the sole anaesthetic medication. Their complication rate was found to be $11.6 \%$ and was most commonly neurologic (3.6\%) and psychological/psychiatric (3.2\%) in origin. ${ }^{[8]}$

Bala et al. recently published a similar study investigating perioperative complications in DBS surgery. ${ }^{[12]}$ Their study included a multi-modal anaesthetic approach with $9(13.8 \%)$ patients receiving general anaesthesia and $56(86.2 \%)$ receiving MAC. Both intra- and post-operative complications were reported, with incidence of $24 \%$ and $15.4 \%$, respectively. Hypertension was responsible for $16.9 \%$ of all reported intra-operative complications. Other reported complications included arrhythmias, venous air embolism or tension pneumocephalus, and post-operative intracranial haemorrhage. Bala et al. reported a significantly higher complication rate than was reported in our study. One obvious reason for this is that periods of hypertension were included in their study as an intra-operative complication. Contrastingly, only periods of refractory hypertension were included as a complication in our study. In addition, in stereotactic surgery, the complication rate is inversely proportional to the experience of the surgeon. Bala et al. commented that their intra-operative complication rate was higher than other reported incidence rates 'probably due to learning curve'. While unknown whether this learning curve was from anaesthesia or surgical standpoint, it can be assumed to be a combination of both as patient inclusion dated back to 2001 when DBS surgery was still in its infancy. A relative strength of our study is that all procedures were done by a single highly experienced surgeon specialising in DBS surgery at a large volume institution.

We should also note that one common side effect of dexmedetomidine infusion is hypotension. Although we did not report hypotension as a complication outcome, we did report on the use of vasopressors and anti-hypertensives throughout these procedures. Quantitative blood pressure values were not reported, and no predetermined cut-offs for hypertension or hypotension were set. Rather, the assumption was made that if the patient was treated with a vasopressor or anti-hypertensive agent they displayed signs of cardiovascular instability that warranted treatment from the anaesthesia provider. The length of hypertensive or hypotensive episodes was not recorded. As noted in our results section, treatment with medications for hypotension was utilised in 70 cases $(40.23 \%)$ while treatment with antihypertensive medications was utilised in 134 cases $(77.01 \%)$. Despite these interventions, none of our patients presented with any cardiac complications 
as defined as intra- or post-operative myocardial infarction, new onset angina, new onset congestive heart failure or refractory systemic arterial hypertension. It is likely that hypotension requiring vasopressor support during these DBS procedures may have been related to dexmedetomidine infusion. However, we cannot confirm this without more controlled studies.

With regard to the hypertension noted in our patient population, we are unable to determine if the cause was related to inadequate sedation, anxiety, pain or pre-existing hypertension. Dexmedetomidine infusion has actually been reported to reduce the required amount of medication for intra-operative hypertension. ${ }^{[10]}$ However, this finding was determined when contrasting patients undergoing DBS procedures with no sedation versus those who received dexmedetomidine infusions. Not surprisingly, patients receiving dexmedetomidine infusions developed less hypertension than those who received no medications. As noted above, intra-operative hypertension during DBS procedures may be related to poor pre-operative control, patient anxiety or discomfort, or secondary to many other intra-operative factors. ${ }^{[1]}$ Proper treatment is critical as hypertension has been associated with an increased risk of intracerebral haemorrhages. ${ }^{[13,14]}$ We found that anti-hypertensives were utilised in the majority of our cases. Most commonly, patients received bolus doses of medications, while a minority received continuous infusions of anti-hypertensives.

Intra-operative neurologic mapping by a neurophysiologist in an awake patient with MER and macrostimulation continues to be paramount in DBS surgery. ${ }^{[1,8]}$ It is still widely unknown the full extent to how anaesthetic drugs influence MER and macrostimulation. This is postulated because the effects of anaesthetic drugs are inhomogeneous across different parts of the brain and may affect neurophysiology monitoring differently in various anatomic locations. ${ }^{[15]}$ To the best of our knowledge, there are no controlled prospective studies solely assessing the effects of dexmedetomidine on MER and macrostimulation. However, there are many retrospective studies and case reports purporting the benefits of dexmedetomidine use, many of which have been previously mentioned. Current literature supports the use of low-dose dexmedetomidine infusion $(0.3-0.6 \mathrm{mcg} / \mathrm{kg} / \mathrm{h})$ with or without a loading dose for monitoring of MER and macrostimulation. ${ }^{[1,10,11]}$ This study also supports this hypothesis as we had a high successful procedure rate with no interference in MER and macrostimulation with the use of dexmedetomidine.

Regardless of our promising results and other supporting research, concern remains regarding the use of dexmedetomidine for DBS and other similar procedures. Previous studies suggest dexmedetomidine may have untoward effects on neurophysiologic monitoring, yet they remain unclear. ${ }^{[16-18]}$ In both our study and that of Rozet et al., brain mapping was not influenced during dexmedetomidine infusion due to the drug's fast washout which allows for optimal recordings. ${ }^{[10]}$ In addition to dexmedetomidine, previous research also supports the use of propofol for these DBS procedures. Although it can be used safely, there are limitations. For example, as patients are treated for hypertension intraoperatively and then must progress to GETA, hypotension on induction is common place and significant with propofol inductions. In addition, Khatib et al. argue that 'due to the complex neuronal circuitry between the striatum and the globus pallidus/substantia nigra, and the globus pallidus externa and subthalamic nucleus where GABA-ergic pathways are involved, sparing use or avoidance of anesthetic medications with GABA-ergic activity like benzodiazepines or propofol during the DBS procedure is advisable. ${ }^{[8]}$ Finally, sneezing is a known complication from propofol, which is documented by the manufacturer. Although typically benign, sneezing during a DBS procedure can lead to difficulty in surgical mapping, increases in intracranial pressure, intra-operative haemorrhage and patient injury while in the surgical head-frame.

As with other studies, our findings need to be considered with our study limitations, which include a retrospective study design and a small sample size. Due to our small sample size, the study could be underpowered to reveal more subtle findings, such as risk factors for complications.

Despite these limitations, our study potentiates current evidence supporting the use of dexmedetomidine for DBS procedures. The unique characteristics of dexmedetomidine make it a valuable option in the anaesthesiologist's armamentarium for the unique demands that present with DBS cases.

\section{Financial support and sponsorship}

Nil.

\section{Conflicts of interest}

There are no conflicts of interest.

\section{REFERENCES}

1. Chakrabarti R, Ghazanwy M, Tewari A. Anesthetic challenges for deep brain stimulation: A systematic approach. N Am J Med Sci 2014;6:359-69.

2. Mayberg HS, Lozano AM, Voon V, McNeely HE, Seminowicz D, Hamani $C$, et al. Deep brain stimulation for treatment-resistant depression. Neuron 2005;45:651-60.

3. Greenberg BD, Malone DA, Friehs GM, Rezai AR, Kubu CS, Malloy PF, et al. Three-year outcomes in deep brain stimulation for highly resistant obsessive-compulsive disorder. Neuropsychopharmacology 2006;31:2384-93.

4. Young RF, Brechner $T$. Electrical stimulation of the 
brain for relief of intractable pain due to cancer. Cancer 1986;57:1266-72.

5. Wu C, Sharan AD. Neurostimulation for the treatment of epilepsy: A review of current surgical interventions. Neuromodulation 2013;16:10-24.

6. Venkatraghavan L, Luciano M, Manninen P. Review article: Anesthetic management of patients undergoing deep brain stimulator insertion. Anesth Analg 2010;110:1138-45.

7. Deiner S, Hagen J. Parkinson's disease and deep brain stimulator placement. Anesthesiol Clin 2009;27:391-415.

8. Khatib R, Ebrahim Z, Rezai A, Cata JP, Boulis NM, John Doyle D, et al. Perioperative events during deep brain stimulation: The experience at cleveland clinic. J Neurosurg Anesthesiol 2008;20:36-40.

9. Precedex: Redefining Sedation, a Clinical Monograph. Chicago: Abbott Laboratories; 2001.

10. Rozet I, Muangman S, Vavilala MS, Lee LA, Souter MJ, Domino KJ, et al. Clinical experience with dexmedetomidine for implantation of deep brain stimulators in Parkinson's disease. Anesth Analg 2006;103:1224-8.

11. Sassi M, Zekaj E, Grotta A, Pollini A, Pellanda A, Borroni M, et al. Safety in the use of dexmedetomidine (precedex) for deep brain stimulation surgery: Our experience in 23 randomized patients. Neuromodulation 2013;16:401-6.

12. Bala R, Chaturvedi A, Pandia MP, Birthal PK. Anaesthetic management and perioperative complications during deep brain stimulation surgery: Our institutional experience. J Neuroanaesth Crit Care 2016;3:119-25.

13. Binder DK, Rau GM, Starr PA. Risk factors for hemorrhage during microelectrode-guided deep brain stimulator implantation for movement disorders. Neurosurgery 2005;56:722-32.

14. Gorgulho A, De Salles AA, Frighetto L, Behnke E. Incidence of hemorrhage associated with electrophysiological studies performed using macroelectrodes and microelectrodes in functional neurosurgery. J Neurosurg 2005;102:888-96.

15. Velly LJ, Rey MF, Bruder NJ, Gouvitsos FA, Witjas T, Regis JM, et al. Differential dynamic of action on cortical and subcortical structures of anesthetic agents during induction of anesthesia. Anesthesiology 2007;107:202-12.

16. Mack PF, Perrine K, Kobylarz E, Schwartz TH, Lien CA. Dexmedetomidine and neurocognitive testing in awake craniotomy. J Neurosurg Anesthesiol 2004;16:20-5.

17. Ard J, Doyle W, Bekker A. Awake craniotomy with dexmedetomidine in pediatric patients. J Neurosurg Anesthesiol 2003;15:263-6.

18. Bustillo MA, Lazar RM, Finck AD, Fitzsimmons B, Berman MF, Pile-Spellman J, et al. Dexmedetomidine may impair cognitive testing during endovascular embolization of cerebral arteriovenous malformations: A retrospective case report series. J Neurosurg Anesthesiol 2002;14:209-12. 